\title{
Characterization of Mayer-wave oscillations in functional near-infrared spectroscopy using a physiologically informed model of the neural power spectra
}

\author{
Robert Luke $\odot$, a,b, $*$ Maureen J. Shader $\odot,{ }^{\text {b,c }}$ and David McAlpine $\odot^{\text {a }}$ \\ ${ }^{a}$ Macquarie University, Australian Hearing Hub, Macquarie University Hearing, \\ Department of Linguistics, Sydney, Australia \\ ${ }^{\mathrm{b}}$ Bionics Institute, Melbourne, Australia \\ ${ }^{c}$ The University of Melbourne, Department of Medical Bionics, Melbourne, Australia
}

\begin{abstract}
Significance: Mayer waves are spontaneous oscillations in arterial blood pressure that can mask cortical hemodynamic responses associated with neural activity of interest.

Aim: We aim to characterize the properties of oscillations in the functional near-infrared spectroscopy (fNIRS) signal generated by Mayer waves in a large sample of fNIRS recordings. Further, we aim to determine the impact of short-channel correction for the attenuation of these unwanted signal components.

Approach: Mayer-wave oscillation parameters were extracted from 310 fNIRS measurements using the fitting oscillations and one-over-f method to compute normative values. The effect of short-channel correction on Mayer-wave oscillation power was quantified on 222 measurements. The practical benefit of the short-channel correction approach for reducing Mayer waves and improving response detection was also evaluated on a subgroup of 17 fNIRS measurements collected during a passive auditory speech detection experiment.
\end{abstract}

Results: Mayer-wave oscillations had a mean frequency of $0.108 \mathrm{~Hz}$, bandwidth of $0.04 \mathrm{~Hz}$, and power of $3.5 \mu \mathrm{M}^{2} / \mathrm{Hz}$. The distribution of oscillation signal power was positively skewed, with some measurements containing large Mayer waves. Short-channel correction significantly reduced the amplitude of these undesired signals; greater attenuation was observed for measurements containing larger Mayer-wave oscillations.

Conclusions: A robust method for quantifying Mayer-wave oscillations in the fNIRS signal spectrum was presented and used to provide normative parameterization. Short-channel correction is recommended as an approach for attenuating Mayer waves, particularly in participants with large oscillations.

(0) The Authors. Published by SPIE under a Creative Commons Attribution 4.0 International License. Distribution or reproduction of this work in whole or in part requires full attribution of the original publication, including its DOI. [DOI: 10.1117/1.NPh.8.4.041001]

Keywords: functional near-infrared spectroscopy; Mayer waves; signal processing.

Paper 21044LRR received Sep. 5, 2021; accepted for publication Nov. 23, 2021; published online Dec. 8, 2021; corrected Dec. 21, 2021.

\section{Introduction}

The ability to explore and understand the brain requires the differentiation of signals that arise from the neural activity of interest from signals that are generated by other factors or non-neural processes. Neuroimaging techniques that estimate the concentration of oxygenated and/or deoxygenated hemoglobin within the cortical blood flow, including functional magnetic resonance imaging and functional near-infrared spectroscopy (fNIRS), are susceptible to disruptions

*Address all correspondence to Robert Luke, robert.luke@mq.edu.au 
from non-neural cardiovascular oscillations in the signal. ${ }^{1}$ For example, oscillations corresponding to heart rate $(\sim 1 \mathrm{~Hz})$ and respiratory rate $(\sim 0.3 \mathrm{~Hz})$ are present within the broad frequency spectrum of the measured signal and can be removed or reduced through filtering. Another cardiovascular component is Mayer waves, which are spontaneous oscillations in arterial blood pressure with a frequency of $\sim 0.1 \mathrm{~Hz}^{2,3}$ Mayer waves are not easily removed from hemodynamic signatures of brain activity as they tend to occur on a time course often confounded with the frequency of a sensory task, for example, and/or the cortical hemodynamic response to that task.

Under the assumption that oscillations in oxygenated hemoglobin originated from the scalp are a product of Mayer waves, Yücel et al. ${ }^{4}$ inserted synthetic hemodynamic response functions (HRFs) into the resting-state recordings from 17 participants in an fNIRS study to demonstrate that large Mayer waves significantly reduce the accuracy of the estimated synthetic hemodynamic response. Further, they demonstrated that utilizing dedicated source-detector pairs for measuring predominantly extra-cerebral activity (8-mm source-detector spacing), referred to as short channels, reduced the impact of Mayer waves on the estimation of the cortical hemodynamic response, and that this effect was more pronounced when the change in the concentration of oxygenated hemoglobin was small relative to the magnitude of the Mayer wave. This confirms that short-channel correction improves the accuracy with which HRFs are estimated for synthetic cortical responses when large Mayer waves are present. However, the effect of shortchannel correction on the characteristics of Mayer waves and the effect of those oscillations on the detection of HRFs in real measurements with event-driven responses has yet to be quantified.

Although many techniques have been proposed to reduce or remove the contribution from Mayer waves to cortical hemodynamic responses, ${ }^{5}$ it is commonly reported that for a small proportion of subjects, HRFs cannot be recovered due to the presence of large-amplitude Mayer waves leading to these participants being excluded from group-level analyses of hemodynamic responses. ${ }^{4}$ A better understanding of how Mayer waves might be quantified will facilitate the development of objective criteria for rejecting hemodynamic responses contaminated by Mayer waves, and in developing and evaluating algorithms to reduce systemic contributions to hemodynamic responses in neuroimaging data.

Mayer-wave activity in fNIRS measurements is typically quantified by aggregating the signal activity within a frequency band centered at $0.1 \mathrm{~Hz}$ and a bandwidth of $0.08 \mathrm{~Hz}$ (e.g., 0.06 to $0.14 \mathrm{~Hz}$ ), although the exact frequency band varies between studies. ${ }^{4,6-8}$ This approach is commonplace across neuroimaging modalities, including EEG, ECOG, and MEG. ${ }^{9}$ However, recent reports suggest that using predefined frequency bands can generate incorrect estimates of oscillatory power, especially if the center frequency of oscillatory activity is intrinsically variable. ${ }^{10}$ Further, using predefined frequency bands conflates changes in the power of oscillations of interest with shifts in center frequency, changes in broadband power, and changes in the non-oscillatory components of neuroimaging signals. ${ }^{11}$ To overcome these shortcomings and facilitate accurate and objective parameterization of oscillatory activity in neuroimaging data, Donoghue et al. ${ }^{12}$ suggested analyzing oscillatory activity by modeling the aperiodic spectral features-decreasing power across increasing frequencies-as well as the periodic/oscillatory components. Rather than ignoring or correcting for these components, which ignores their possible physiological correlates, the algorithm employed by Donoghue et al. ${ }^{12}$ provides a physiologically informed model of the neural power spectra by modeling both distinct functional processes. This approach, called "fitting oscillations and one-over-f" (FOOOF) and provided by the authors as open-source software, allows for the identification of oscillatory activity in hemodynamic signals without the requirement of predefined and specific frequency bands of interest. It is, therefore, well suited for identifying and parameterizing Mayer-wave activity in fNIRS measurements.

Here we quantify the typical frequency and power distribution of Mayer waves from over 300 fNIRS recordings using the FOOOF algorithm. This accords with the approach of Yücel et al., ${ }^{4}$ who utilized synthetic data to demonstrate the ground truth of the effect of short-channel correction as a function of the amplitude of HRFs and Mayer waves. Short-separation channels are optode pairs with a distance typically less than $1 \mathrm{~cm}$ that predominantly measure systemic rather than neural activity. ${ }^{13-17}$ The mitigation effect of using short-separation channel regression for reducing contamination of hemodynamic responses by Mayer waves is evaluated on over 200 
Luke, Shader, and McAlpine: Characterization of Mayer-wave oscillations in functional near-infrared...

fNIRS recordings. In addition, the impact of Mayer waves on the detection of auditory-evoked hemodynamic responses, which typically comprise small responses and poor signal-to-noise ratios, ${ }^{18}$ is evaluated in a subgroup of recordings from 17 participants.

\section{Methods}

\subsection{Data}

The data used in this study were aggregated from a variety of research studies conducted between 2018 and 2021. Measurements were obtained under the Macquarie University Ethics Application References 52020640814625 and 5201500948. In total, 310 measurements are included in this dataset. The studies contained a variety of optode-placement montages, sample rates, experimental designs, tasks, and stimuli. As such, it is not appropriate to compare taskevoked responses across measurements. However, a comparison of systemic oscillations within the signal, which are unrelated to the task-evoked aspect of the data (e.g., Mayer waves), is valid.

Although the dataset of 310 measurements contained a variety of experimental designs, all datasets met a minimum criterion for inclusion in this study. All measurements included a stimulus, in which the repetition rate of the stimulus was never a multiple of $10 \mathrm{~s}$, as this would produce a frequency component indistinguishable from the Mayer wave in the data. Further, all measurements included a randomized interstimulus interval. Although the exact range of interstimulus interval duration varied slightly between experiments, the minimum interval jitter across the dataset was $10 \mathrm{~s}$ and the maximum was $30 \mathrm{~s}$. Similarly, the task repetition rate was not a multiple of $10 \mathrm{~s}$ in any measurement.

All participants were seated in a sound-attenuating booth in a comfortable chair for the duration of the experiments. NIRS data were recorded using a continuous-wave NIRx NIRScoutX device with avalanche photodiode detectors. Measurements were obtained with between 8 and 16 sources and detectors. Experiments lasted between 15 and $80 \mathrm{~min}$. All data were stored in BIDS data format. ${ }^{19}$ Data were processed with MNE-Python, ${ }^{20,21}$ MNE-NIRS, ${ }^{18}$ and Nilearn. ${ }^{22,23}$

\subsection{Quantification of Mayer-Wave Parameters}

The FOOOF method of Donoghue et al. ${ }^{12}$ was employed to quantify oscillations in the fNIRS signal associated with Mayer waves. This method was designed to extract oscillations in neural signals while accounting for the natural structure of the frequency spectrum of neuroimaging signals. As such, it is well suited for quantifying Mayer waves; the method extracts peaks in the power spectra without the need to predefine analysis parameters, such as the signal center frequency or bandwidth.

To quantify the parameters of Mayer waves, a minimal processing pipeline was applied to convert the raw data to oxyhemoglobin concentration. Specifically, the raw data were first converted to optical density. Channels (both long and short) with a scalp coupling index $<0.7$ were excluded from further analysis. ${ }^{24}$ The scalp coupling index was computed using a frequency range of 0.5 to $1.2 \mathrm{~Hz}$. Next the data were downsampled to $1.7 \mathrm{~Hz}$. The data were then converted to hemoglobin concentration using the modified Beer-Lambert law with a partial pathlength factor of $0.1 .^{25-27}$ Channels with a source-detector distance of less than 1.5 or greater than $4.5 \mathrm{~cm}$ were excluded from further analysis. Finally, as the oxyhemoglobin measurement contains a greater contribution from Mayer-wave signals than deoxyhemoglobin, ${ }^{8}$ only the oxyhemoglobin signal was retained for further analysis.

The power spectral-density was then calculated for each channel using the Welch method with a Hamming window comprising 300 samples and 150 samples of overlap, and the spectra averaged across all channels per measurement to generate a single-power spectral-density estimate per recording (in units of $\mu \mathrm{M}^{2} / \mathrm{Hz}$ ). These spectra were then passed to the FOOOF software. The FOOOF software returns estimates of the aperiodic component and oscillations in the signal; if multiple oscillations are detected (e.g., respiratory rate) then the oscillation closest to $0.1 \mathrm{~Hz}$ is retained for further analysis as it is assumed to contain a Mayer-wave component. The output of this analysis is a single estimate of the oscillations present in a recording. Utilizing the software provided by the FOOOF authors is recommended, as it reports the oscillations within 
the likely Mayer-wave range in terms of their center frequency in $\mathrm{Hz}$, bandwidth in $\mathrm{Hz}$, and log power in arbitrary units.

\subsection{Effect of Short-Channel Correction}

We evaluated a subset of 222 files that contained data from short-separation channels to quantify the effect of systemic signal attenuation on the presence of Mayer-wave oscillations in the fNIRS signal. After estimating Mayer-wave parameters when no attenuation was applied to the systemic signal (see Sec. 2.2), we employed the same approach with the additional step of reducing the systemic component of the hemodynamic response through the assessment of activity in short channels being applied after resampling. Many methods have been proposed for attenuating systemic components. This study utilized the method of short-channel regression based on the nearest short channel as described in Sec. 2.1.6 of Scholkmann et al. ${ }^{16}$ (and implementation as provided by NIRx). ${ }^{14,16}$ As a result, the parameters of Mayer-wave oscillations (center frequency, bandwidth, and power) were estimated with and without short-channel-based correction of systemic signals in the hemodynamic response.

A Bland-Altman analysis ${ }^{28}$ was used to determine whether short-channel correction affected Mayer waves differentially as a function of their amplitude. The bootstrapping approach of Ho et al. ${ }^{29}$ was used to compare the magnitude of Mayer-wave oscillations with and without short-channel correction.

\subsection{Single Dataset Example}

To demonstrate the practical utility of measuring the parameters of Mayer waves, a single, publicly available dataset of 17 participants was analyzed. ${ }^{18}$ Participants were exposed to stimuli in a block design protocol but were not asked to actively respond to any stimulus. Each speech stimulus was $5.25 \mathrm{~s}$ in duration, blocks were separated by a random interstimulus interval that was randomly selected from a uniform distribution in the range of 10 to $20 \mathrm{~s}$, a complete protocol description is provided with the source of the openly available dataset by Luke et al. ${ }^{18}$ The presence of an evoked response to auditory-speech stimuli was quantified at an individual level to determine whether data from participants with larger Mayer waves benefited from correction of systemic components in their hemodynamic responses. Mayer waves were quantified for the data from each participant as described in Sec. 2.2.

The data were analyzed using a generalized linear model (GLM), as, due to the statistical properties of the noise in fNIRS signals, ${ }^{30}$ this is suggested to be more appropriate than averaging the data. The data were analyzed as described in Ref. 18. Briefly, the raw data were first converted to optical density and then to hemoglobin concentration using the modified BeerLambert Law with a differential pathlength factor of 0.1 . They were then downsampled to $0.6 \mathrm{~Hz}$, and a GLM was fitted to all channels using a 3-s boxcar function convolved with a glover model. ${ }^{18,22,31} \mathrm{~A}$ cosine drift was included with components up to $0.01 \mathrm{~Hz}$. The fit was performed with a prewhitening procedure using a first-order auto regressive model. Two analyses were performed, one including the mean of the short channels as a regressor in the GLM, and one without any short-channel regressor. Channels over the left and right superior temporal gyri, comprising typical auditory regions of interest, were then combined into a single region of interest using a weighted average of the GLM estimates. ${ }^{32}$ If the $p$ value of the regressor component of the speech task in the GLM estimate was $<0.05$ (i.e., a significant $\mathrm{HbO}$ response evoked by auditory speech), a response was deemed present. As such, for each participant, a task-locked neural response was quantified as either present or absent for both analyses, with and without short-channel regressions.

\section{Results and Discussion}

Accurate estimation of neuroimaging signal components is essential for precise measurements of brain activity. Here we investigate the use of the FOOOF method for characterizing Mayer-wave oscillations in the fNIRS signal. The FOOOF method estimates oscillatory activity in the neuroimaging measurement that is not a component generated by the aperiodic structure of the signal. 


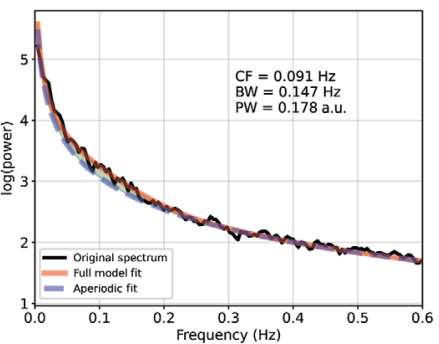

(a)

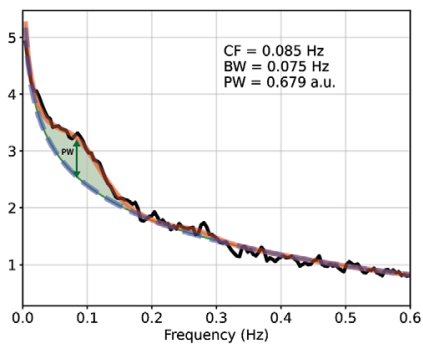

(b)

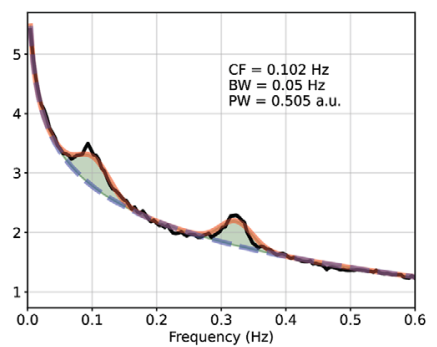

(c)

Fig. 1 Examples of FOOOF fit of fNIRS data. The black line represents the power spectral density of the signal, the red line represents the complete model fit of the FOOOF algorithm, with the blue dashed line indicating the aperiodic portion of the signal, and green-shaded regions marking oscillations in the signal as peaks rising above the aperiodic component. Three examples are provided to demonstrate the appropriateness of the algorithm to fNIRS data in different situations. (a) An example of a measurement with very small Mayer-wave oscillation. (b) An oscillation that is not centered at the expected $0.1 \mathrm{~Hz}$ frequency. (c) A measurement with additional substantial oscillatory activity at $0.32 \mathrm{~Hz}$ which likely represents the breathing/respiratory rate. $\mathrm{CF}$, center frequency $(\mathrm{Hz})$; PW, power estimation (a.u.); and BW, bandwidth $(\mathrm{Hz})$.

Thus characterization of the peaks in the power spectrum is possible without requiring predefined bandwidths. Figure 1 illustrates several representative fits provided by the FOOOF algorithm applied to fNIRS data. The algorithm appropriately models the data in the presence of small [Fig. 1(a)] and large [Fig. 1(b)] oscillation peaks, for oscillations not centered exactly at $0.1 \mathrm{~Hz}$ [Fig. 1(b)], and in the presence of additional oscillations in the data (likely respiratory rate) [Fig. 1(c)].

The underlying code to run the FOOOF algorithm is available at the original authors' website, ${ }^{12}$ and a convenient wrapper to run the analysis with fNIRS data is provided as part of the MNE-NIRS package. ${ }^{18}$

\subsection{Quantification of Mayer-Wave Parameters on a Cohort of 310 Measurements}

The FOOOF algorithm was applied to a cohort of 310 measurements. An oscillatory component was detected in all but four measurements. The mean center frequency of the oscillations was $0.108 \mathrm{~Hz}$ and the median value was $0.097 \mathrm{~Hz}$ [see Fig. 2(a)]. This was expected as the analysis approach actively selected the frequency component closest to $0.1 \mathrm{~Hz}$ in instances when more than one oscillatory peak was identified in the signal. To confirm that the selected peaks were

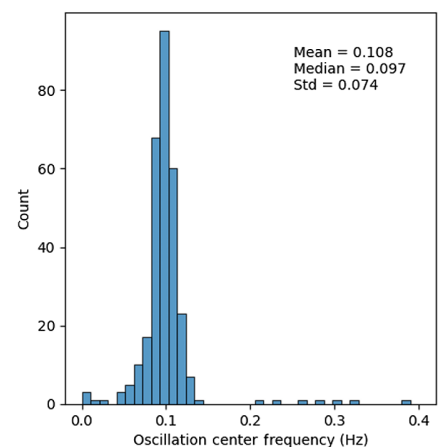

(a)

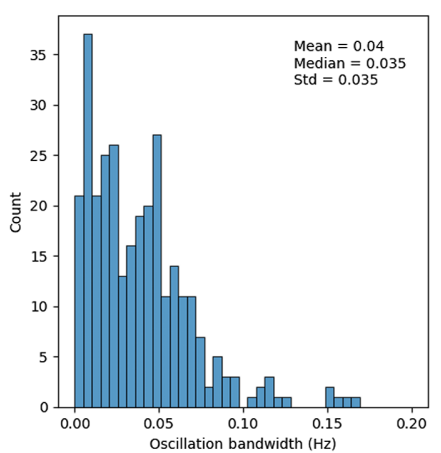

(b)

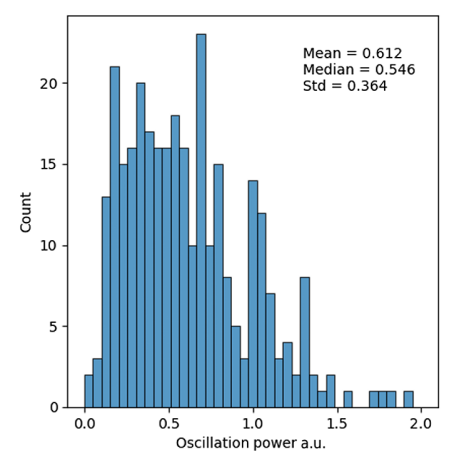

(c)

Fig. 2 Quantification of Mayer-wave parameters: (a) center frequency of oscillation components, (b) oscillation bandwidth, and (c) power of oscillation component. Note that the power of Mayer waves is not normally distributed, with some participants having much larger values than the group majority. 
indeed related to Mayer-wave activity, the model was rerun while retaining oscillations with frequencies closest to typical cutoff frequencies used in frequency-band aggregates of Mayer waves, including 0.05 and $0.15 \mathrm{~Hz}$. With these modified parameters, the median frequency of the resulting oscillatory component was essentially unchanged at 0.105 and $0.109 \mathrm{~Hz}$ for the lower and upper cutoff frequencies, respectively, demonstrating that the model was fitting robust oscillatory components in the data related to Mayer-wave activity.

The bandwidth of the oscillations was highly non-normally distributed with a median value of $0.035 \mathrm{~Hz}$ [Fig. 2(b)], corresponding to a typical Mayer-wave frequency range of $\sim 0.065$ to $0.135 \mathrm{~Hz}$. Similarly, Mayer-wave power was not uniformly distributed across participants [Fig. 2(c)]. Instead, the median log power of oscillations was 0.55 a.u., and the distribution was positively skewed. This corresponds to an oscillatory component in the fNIRS signal of $3.5 \mu \mathrm{M}^{2} / \mathrm{Hz}$. Nineteen of 310 measurements $(6 \%)$ contained Mayer waves that were 2 standard deviations above the median oscillation power, largely consistent with anecdotal reports of a small proportion of participants having particularly large Mayer waves. Unbiased quantification of the parameters of these oscillations while including the aperiodic component in the spectra will assist in the development of algorithms for identifying and attenuating these unwanted signals in hemodynamic responses.

These results demonstrate the added utility of the FOOOF method over a simple band-pass power metric. In addition to extracting oscillation power, the proposed technique also quantifies the oscillation center frequency and bandwidth. Although the average values for each metric are in line with expectations from the previous studies, the variability of values demonstrates that further research is required into the underlying cause of the variation and interaction between each characteristic of the Mayer-wave signals. For example, research using a band-pass power metric has shown that the magnitude of Mayer waves varies across the scalp, ${ }^{8}$ yet as demonstrated by Donoghue et al., ${ }^{12}$ this may be caused by either a true variation in oscillation power or confounding variation in another characteristic such as bandwidth. This study included a wide range of sensor positions and experimental designs to demonstrate that the algorithm works for a variety of data, and also to demonstrate the variability in Mayer-wave characteristics. We hope that this new tool will facilitate research into variation in Mayer-wave oscillations across various factors such as scalp position, participant state, and experimental paradigm.

\subsection{Effect of Short-Channel Correction on Mayer Waves}

Next, the degree to which the power of Mayer waves is attenuated following short-channel correction was evaluated, and it was assessed if any characteristic of the uncorrected Mayer-wave oscillations (i.e., greater oscillatory power) was associated with larger power attenuation after correction. Of the 310 recordings in the dataset, 222 contained short-channel data, providing a means by which to assess the effect of short-channel correction on Mayer-wave power. These measurements were reanalyzed to quantify the center frequency, bandwidth, and power of the Mayer waves after short-channel systemic correction was applied to the long-channel data.

Applying short-channel correction did not significantly alter the frequency distribution or bandwidth of Mayer-wave components in the signal [Fig. 3(a)]. A small but significant reduction in the signal power of Mayer waves was apparent when short-channel systemic correction was applied to the entire dataset (effect $=-0.0459,95 \% \mathrm{CI}[-0.066,-0.027], N=222, p<0.001$ ).

The reduction in Mayer-wave power was more pronounced in measurements with a large systemic component in the uncorrected data. Bland-Altman analysis [Fig. 3(b)] demonstrates that the difference in oscillation power when the correction is applied varies as a function of oscillation power. As such, we analyzed the measurements with the largest Mayer-wave power using a median split to divide the dataset into two groups. The group with larger Mayer waves (107 measurements) showed a reduction of 0.11 in log power when short-channel correction was applied (effect $=-0.11,95 \% \mathrm{CI}[-0.136,-0.086], N=107, p<0.001$ ), whereas the group with smaller Mayer-wave oscillations showed no consistent change in oscillation power $\mathrm{f}$ ollowing short-channel correction (effect $=-0.0018,95 \% \mathrm{CI}[-0.0145,0.021], N=115$, $p=0.841)$. On this basis, short-channel correction to reduce Mayer-wave components in hemodynamic signals provides significant benefit when Mayer-wave components are relatively large, supporting the HRF modeling of Yücel et al. ${ }^{4}$ Further, short-channel correction does not 


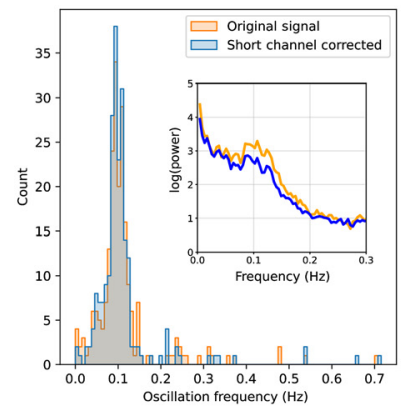

(a)

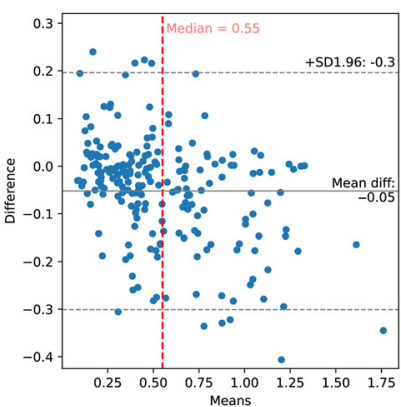

(b)

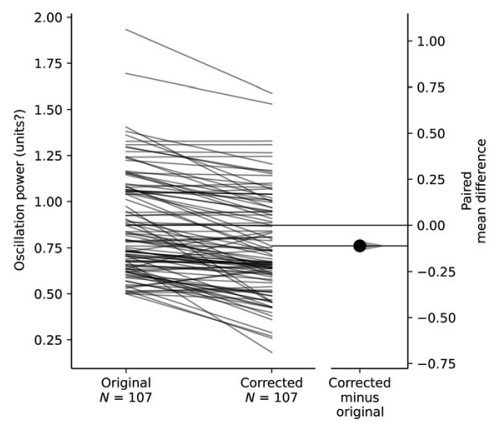

(c)

Fig. 3 Effect of short-channel correction on the power of Mayer-wave oscillations. (a) Distribution of frequency component of Mayer waves with and without short-channel correction applied. Inset illustrates an example measurement with and without short-channel correction. Note that the increase in power around $0.1 \mathrm{~Hz}$ is reduced when correction is applied. (b) Bland-Altman plot illustrating the difference in Mayer-wave power when short-channel correction is applied as a factor of the oscillation power. (c) Paired comparison illustrating the effect of short-channel correction on oscillation power. Note that short-channel correction does not affect the frequency of the oscillation component but does reduce the power of the oscillation, particularly for measurements containing a large Mayer-wave oscillatory component.

systematically alter the oscillation component when applied to measurements with small Mayer waves, indicating that short-channel systemic component correction can be broadly applied to fNIRS measurements without unwanted signal distortion.

\subsection{Effect of Short-Channel Correction on the Amplitude of Evoked Responses}

Systemic noise can mask the presence of neural responses in fNIRS measurements. Accounting for data obtained from short channels in the signal processing pipeline may mitigate unwanted systemic components and improve the detection of neural responses. As such, we analyzed the measurements from a publicly available dataset ${ }^{18}$ comprising fNIRS data of 17 participants, to determine if measurements with large Mayer waves particularly benefited from short-channel correction.

As for the larger data set (Sec. 3.2), data were divided into two groups using a median split of the oscillation power in Mayer waves. Without short-channel regression, just two of nine measurements with the largest Mayer waves were observed to contain significant evoked responses to auditory-speech stimuli. When short-channel regression was employed, however, this increased to eight of nine measurements, suggesting that short-channel correction does indeed improve the detection of auditory-evoked cortical responses in participants with large Mayer waves. These data were collected with an experimental design with randomized interstimulus interval times to minimize listener expectation and mitigate the effect of Mayer-wave contributions. Even greater benefits from short-channel correction may be observed for data collected without randomized interstimulus intervals.

\section{Conclusion}

A method to quantify the presence of Mayer-wave oscillations in fNIRS measurements was introduced based on the well-established and publicly available algorithm of Donoghue et al. ${ }^{12}$ The technique was applied to a dataset of 310 fNIRS measurements, and the population normative values are reported. The distribution of oscillation power in Mayer waves was found to be positively skewed. From a subset of 222 recordings, it was demonstrated that applying short-channel correction significantly reduces contamination by Mayer-wave oscillations in responses containing large oscillatory activity but does not consistently modify the oscillation properties of responses with smaller Mayer waves. The applicability of this technique for 
quantifying Mayer-wave oscillations in an evoked-response experiment was demonstrated on a publicly available dataset. From this, we recommend using the FOOOF method to quantify Mayer-wave oscillatory activity and to evaluate the efficacy of algorithms designed to mitigate systemic components in hemodynamic responses, including through the use of short-channelbased algorithms when assessing hemodynamic responses generated using fNIRS.

\section{Disclosures}

The authors have no conflicts of interest to declare.

\section{Acknowledgments}

This study was supported by an Australian Research Council Laureate Fellowship (No. FL160100108) awarded to David McAlpine.

\section{Code, Data, and Materials Availability}

The FOOOF method is available at the website [https://fooof-tools.github.io/fooof/]. ${ }^{12}$ An implementation of the algorithm for use with fNIRS data is available at the MNE-NIRS website.

\section{References}

1. Y. Tong et al., "Low-frequency oscillations measured in the periphery with near-infrared spectroscopy are strongly correlated with blood oxygen level-dependent functional magnetic resonance imaging signals," J. Biomed. Opt. 17, 106004 (2012).

2. M. G. Ghali and G. Z. Ghali, "Mechanisms contributing to the generation of Mayer waves," Front. Neurosci. 14, 395 (2020).

3. C. Julien, "The enigma of Mayer waves: facts and models," Cardiovasc. Res. 70, 12-21 (2006).

4. M. A. Yücel et al., "Mayer waves reduce the accuracy of estimated hemodynamic response functions in functional near-infrared spectroscopy," Biomed. Opt. Express 7, 3078-3088 (2016).

5. A. von Lühmann et al., "Improved physiological noise regression in fNIRS: a multimodal extension of the general linear model using temporally embedded canonical correlation analysis," Neuroimage 208, 116472 (2020).

6. E. Kirilina et al., "Identifying and quantifying main components of physiological noise in functional near infrared spectroscopy on the prefrontal cortex," Front. Hum. Neurosci. 7, 864 (2013).

7. P. Pinti et al., "Current status and issues regarding pre-processing of fNIRS neuroimaging data: an investigation of diverse signal filtering methods within a general linear model framework," Front. Hum. Neurosci. 12, 505 (2019).

8. Y. Zhang et al., "Multiregional functional near-infrared spectroscopy reveals globally symmetrical and frequency-specific patterns of superficial interference," Biomed. Opt. Express 6, 2786-2802 (2015).

9. H. O. de Beeck and C. Nakatani, Introduction to Human Neuroimaging, Cambridge University Press (2019).

10. M. M. Lansbergen et al., "The increase in theta/beta ratio on resting-state EEG in boys with attention-deficit/hyperactivity disorder is mediated by slow alpha peak frequency," Prog. Neuro-Psychopharmacol. Biol. Psychiatr. 35, 47-52 (2011).

11. T. Donoghue, J. Dominguez, and B. Voytek, "Electrophysiological frequency band ratio measures conflate periodic and aperiodic neural activity," eNeuro 7, ENEURO.019220.2020 (2020).

12. T. Donoghue et al., "Parameterizing neural power spectra into periodic and aperiodic components," Nat. Neurosci. 23, 1655-1665 (2020). 
Luke, Shader, and McAlpine: Characterization of Mayer-wave oscillations in functional near-infrared...

13. F. Fabbri et al., "Optical measurements of absorption changes in two-layered diffusive media," Phys. Med. Biol. 49, 1183-1201 (2004).

14. R. B. Saager and A. J. Berger, "Direct characterization and removal of interfering absorption trends in two-layer turbid media," J. Opt. Soc. Am. 22, 1874-1882 (2005).

15. H. Santosa et al., "Quantitative comparison of correction techniques for removing systemic physiological signal in functional near-infrared spectroscopy studies," Neurophotonics 7, 035009 (2020).

16. F. Scholkmann, A. J. Metz, and M. Wolf, "Measuring tissue hemodynamics and oxygenation by continuous-wave functional near-infrared spectroscopy: how robust are the different calculation methods against movement artifacts?" Physiol. Meas. 35, 717-734 (2014).

17. D. Wyser et al., "Short-channel regression in functional near-infrared spectroscopy is more effective when considering heterogeneous scalp hemodynamics," Neurophotonics 7, 035011 (2020).

18. R. Luke et al., "Analysis methods for measuring passive auditory fNIRS responses generated by a block-design paradigm," Neurophotonics 8, 025008 (2021).

19. K. J. Gorgolewski et al., "The brain imaging data structure, a format for organizing and describing outputs of neuroimaging experiments," Sci. Data 3, 1-9 (2016).

20. A. Gramfort et al., "MEG and EEG data analysis with MNE-Python," Front. Neurosci. 7, 267 (2013).

21. A. Gramfort et al., "MNE software for processing MEG and EEG data," Neuroimage 86, 446-460 (2014).

22. A. Abraham et al., "Machine learning for neuroimaging with scikit-learn," Front. Neuroinf. 8, 14 (2014).

23. B. Thirion et al., "Expanding the Nilearn vision: machine learning and statistics for fMRI in Python," in Organization Human Brain Mapping Annu. Meeting (2021).

24. L. Pollonini et al., "Auditory cortex activation to natural speech and simulated cochlear implant speech measured with functional near-infrared spectroscopy," Hear. Res. 309, 84-93 (2014).

25. C. W. Hoppes et al., "Changes in cortical activation during dual-task walking in individuals with and without visual vertigo," J. Neurol. Phys. Ther. 44, 156 (2020).

26. H. Santosa et al., "The NIRS Brain AnalyzIR toolbox," Algorithms 11, 73 (2018).

27. G. Strangman, M. A. Franceschini, and D. A. Boas, "Factors affecting the accuracy of nearinfrared spectroscopy concentration calculations for focal changes in oxygenation parameters," Neuroimage 18, 865-879 (2003).

28. D. G. Altman and J. M. Bland, "Measurement in medicine: the analysis of method comparison studies," J. R. Stat. Soc. Ser. D 32, 307-317 (1983).

29. J. Ho et al., "Moving beyond $P$ values: data analysis with estimation graphics," Nat. Methods 16, 565-566 (2019).

30. T. J. Huppert, "Commentary on the statistical properties of noise and its implication on general linear models in functional near-infrared spectroscopy," Neurophotonics $\mathbf{3}, 010401$ (2016).

31. G. H. Glover, "Deconvolution of impulse response in event-related BOLD fMRI," Neuroimage 9, 416-429 (1999).

32. M. J. Shader et al., "The use of broad vs restricted regions of interest in functional nearinfrared spectroscopy for measuring cortical activation to auditory-only and visual-only speech," Hear. Res. 406, 108256 (2021). 